\title{
RELIABILITY ANALYSIS OF THE UNCERTAIN FRACTIONAL-ORDER DYNAMIC SYSTEM WITH STATE CONSTRAINT
}

\author{
Ting Jin ${ }^{1}$, Hongxuan Xia ${ }^{1}$, and Shangce $\mathrm{Gao}^{2}$ \\ ${ }^{1}$ Nanjing Forestry University \\ ${ }^{2}$ University of Toyama
}

April 18, 2021

\begin{abstract}
Uncertain fractional-order differential equations driven by Liu process are of significance to depict the heredity and memory features of uncertain dynamical systems. This paper primarily investigates the reliability analysis of the uncertain fractionalorder dynamic system with a state constraint. On the basis of the first-hitting time (FHT), a novel uncertain fractional-order dynamic system considering a state constraint is proposed. Secondly, in view of the relation between the initial state and the required standard, such uncertain fractional-order dynamic systems are subdivided into four types. The concept of reliability of proposed uncertain system with a state constraint is presented innovatively. Corresponding reliability indexes are ulteriorly formulated via FHT theorems. Lastly, the uncertain fractional-order dynamic system with a state constraint is applied to different physical and financial dynamical models. The analytic expression of the reliability index is derived to demonstrate the reasonableness of our model. Meanwhile, expected time response and American barrier option prices are calculated by using the predictor-corrector scheme. A sensitivity analysis is also illustrated with respect to various conditions.
\end{abstract}

\section{Hosted file}

Reliabilityanalysis0404.pdf available at https://authorea.com/users/408527/articles/518504reliability-analysis-of-the-uncertain-fractional-order-dynamic-system-with-stateconstraint 
figures/E-T1/E-T1-eps-converted-to.pdf 
figures/E-T2/E-T2-eps-converted-to.pdf 